\title{
Computational and experimental analysis of the churning power losses in an industrial planetary speed reducer
}

\author{
F. Concli \& C. Gorla \\ Politecnico di Milano, Dipartimento di Meccanica, Milan, Italy
}

\begin{abstract}
Advantages of planetary speed reducers are well known and due to their compact design and power density they are suitable for a wide range of applications. Efficiency is becoming more and more of a main concern in the design of power transmissions and the demand for high efficiency gearboxes is continuously increasing. For this reason it is important to have some models in order to quantify the power losses of those already during the design stage. Some theoretical or semi-empirical models that allow us to estimate losses like those of bearings, of seals, of gear meshing (due to sliding) and, for ordinary gears, those of churning are available in literature. In the case of planetary speed reducers, where the motion of the planet carrier causes a rotatory motion of the planets around the axis of the gearbox, the oil splash lubrication is an important source of losses. This report introduces a multiphase CFD model for the prediction of the churning losses characteristic of planetary gears. The analysis has been carried out by means of an unsteady Volume of Fluid (VOF) model and implemented on a commercial software (Fluent). The whole geometry of the speed reducer and many operating conditions like the rotational speed, the oil level and the operating temperature have been taken into account. Moreover the results of an experimental testing campaign on an especially designed gearbox are presented and compared with the computation ones in order to validate the model. The two approaches give results in good agreement.
\end{abstract}

Keywords: planetary speed reducers, churning losses, multiphase flow simulation, VOF. 


\section{Introduction}

In the last years, efficiency is becoming more and more a main concern also in the design of power transmissions and the demand for high efficiency gearboxes is continuously increasing. Just having appropriate models to predict the final efficiency of the speed reducer [1] it is possible to take the correct choices since the design step, avoiding waste of time and money. Some models for the estimation of the different sources of losses like those of bearings [2], of seals [3], of gear meshing (due to sliding) [3] and, for ordinary gears, those of churning [4] already exist. What is still missing, is an appropriate model for the prediction of the churning losses in planetary speed reducers. In this kind of gearing, in fact, the motion of the planetary gears due to the planet carrier rotation is an additional source of losses.

Aim of this study is to provide a model for the correct estimation of this kind of losses and, consequently, for the correct estimation of the efficiency of the whole transmission. The model has been performed by mean of a computational fluid dynamic (CFD) analysis that simulates the behaviour of the air-oil lubricant mixture.

To validate the numerical model, an industrial planetary speed reducer has been conveniently modified in order to be able to measure the churning losses alone caused by the motion of the planetary gears. This gearbox has been tested on an especially designed test rig.

\section{Problem definition}

Figure 1 shows an example of a planetary gearing: it can be seen the sun gear, the planetary gears, the planet carrier and the external crown.

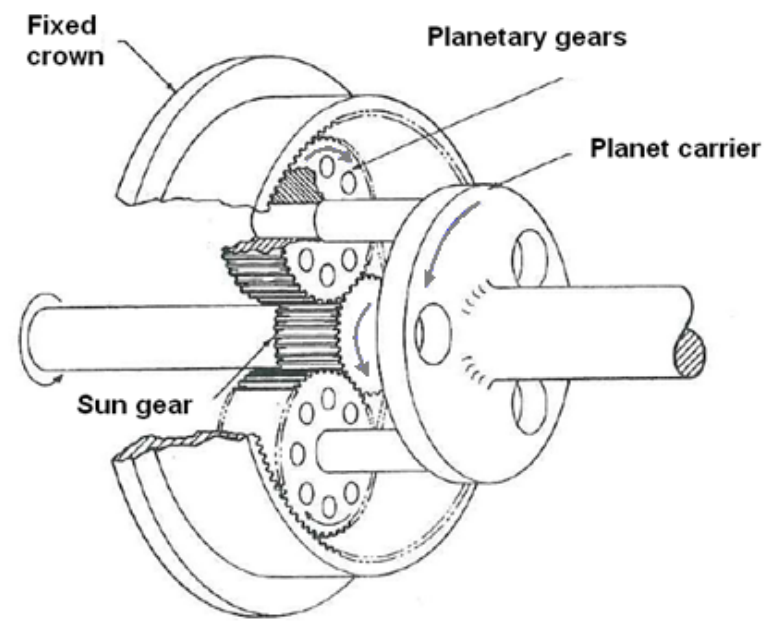

Figure 1: $\quad$ Planetary gearing. 
In the common configuration, the power flows from the sun gear shaft to the planet carrier shaft. The planetary gears have therefore two rotatory motion components: the first one is a rotation around their axis and the second one is a motion with a circular path around the gearbox axis due to the planet carrier rotation on which they are mounted.

The planetary speed reducers are generally oil splash lubricated and it is just the interaction between the rotating elements and the lubricant air-oil mixture the source of losses investigated by the presented model.

The influence parameters are the oil level and its properties, such as the viscosity and the density (functions of the temperature), the geometry and, of course, the rotational speed. The transmitted torque influences this kind of losses indirectly: increasing the transmitted torque means more load dependent power losses (like the meshing ones and part of the bearing ones) and, consequently, a higher regime temperature of the lubricant.

\section{Geometry modifications}

The initial geometry of the analysed speed reducer is shown in figure 2 .

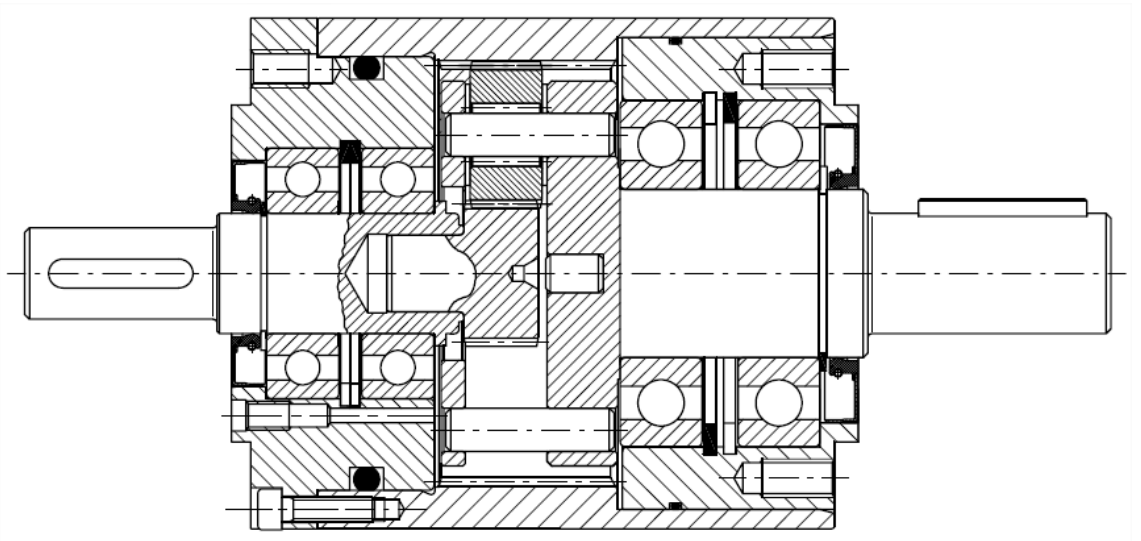

Figure 2: $\quad$ Initial geometry of the speed reducer.

The speed reducer is composed by a sun gear, 3 planetary gears and a "double disk" planet carrier. Both, the input and the output shaft, are mounted by mean of 2 bearings. Two contact seal are also present to avoid leakage of lubricant. In order to evaluate the churning power losses related to the motion of the planetary gears around the gearbox axis due to the rotation of the planer carrier only, the originary geometry of the speed reducer has been modified. The teeth of the external crown have been removed by machining and the sun gear has been substituted with a smaller and cylindrical (without teeth) component. The aim of these modifications is to prevent the engaging of the gears, avoiding sliding losses and churning losses due to the rotation of the gears around their axis. This modified geometry has been used both for the numerical model and the experimental testing campaign. 


\section{Model set up}

\subsection{Geometry and mesh}

The domain for the CFD analysis is the internal free volume of the speed reducer after the above described geometry modifications. Figure 3 shows this volume marked in grey.
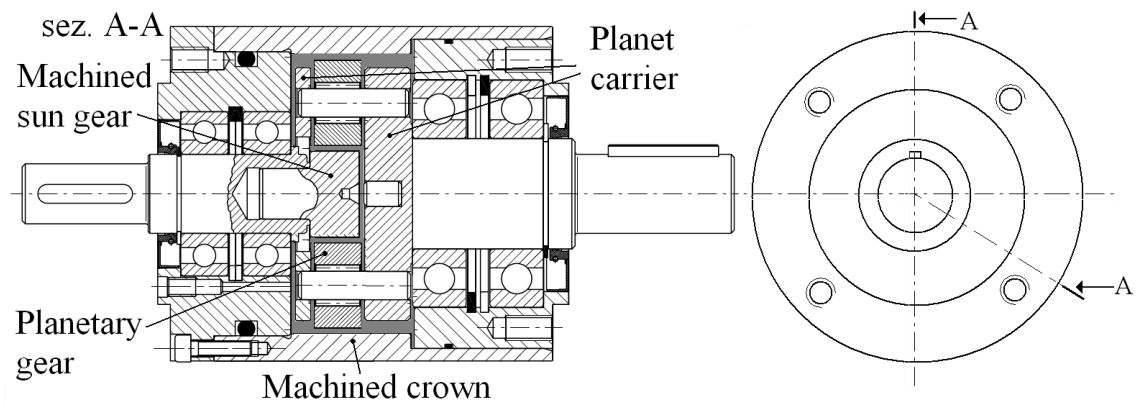

Figure 3: Modified geometry of the speed reducer: the computational domain is marked in yellow.

The computational domain for the CFD analysis has been modelled by means of $3 \mathrm{D}$ cad software and discretized with a swept mesh. This meshing technique consists in creating a mesh on one side of the region, known as the source side, and then copying the nodes of that mesh, one element layer at a time, until the final side, known as the target side, is reached.

The whole model has been discretized with about 500000 hexahedral cells. This kind of elements allows a larger aspect ratio compared with the tetrahedral cells in which it will invariably affects the skewness of the cell, which is undesirable as it may impede accuracy and convergence.

\subsection{Parameter setting}

A VOF multiphase approach has been chosen for the analysis. The VOF formulation relies on the fact that two or more fluids (or phases) are not interpenetrating. The fields for all variables and properties are shared by the phases and represent volume-averaged values, as long as the volume fraction of each of the phases is known at each location. Thus the variables and properties in any given cell are either purely representative of one of the phases, or representative of a mixture of the phases, depending upon the volume fraction values. The tracking of the interface between the phases is accomplished by the solution of a continuity equation for the volume fraction of one of the phases

$$
\frac{\partial}{\partial t}\left(\alpha_{q} \rho_{q}\right)+\nabla \cdot\left(\alpha_{q} \rho_{q} \vec{v}_{q}\right)=S_{\alpha_{q}}+\sum_{p=1}^{n}\left(\dot{m}_{p q}-\dot{m}_{q p}\right)
$$

where $\rho_{q}$ is the density of the $q^{\text {th }}$ phase, $\alpha_{q}$ the volume fraction of that phase, $\dot{m}_{p q}$ and $\dot{m}_{q p}$ the mass transfer from phase $q$ to phase $p$ and vice versa 
respectively. This volume fraction equation has been solved be mean of an explicit scheme discretisation in order to avoid the numerical diffusion. That means solving

$$
\frac{\alpha_{q}^{n+1} \rho_{q}^{n+1}-\alpha_{q}^{n} \rho_{q}^{n}}{\Delta t} V+\sum_{f}\left(\rho_{q} U_{f}^{n} \alpha_{q, f}^{n}\right)=\left[S_{\alpha_{q}}+\sum_{p=1}^{n}\left(\dot{m}_{p q}-\dot{m}_{q p}\right)\right] V
$$

where $n+1$ and $n$ are the indexes for the new time step and the preious one, $\alpha_{q, f}^{n}$ is the face value of the $q^{t h}$ volume fraction computed with the GeoReconstruction scheme, $V$ is the volume of the cell and $U_{f}^{n}$ is the volume flux on the face. The Geo-Reconstruction approach is an accurate scheme that assumes that the interface between two fluids has a linear slope within each cell, and uses this linear shape for the calculation of the advection of fluid through the cell faces. This scheme avoids the numerical diffusion but needs an accurate grid.

The properties appearing in the above transport equations are determined by the presence of the component phases in each control volume. In a two-phase system, if the phases are represented, for example, by the subscripts air and oil, and if the volume fraction of the second of these is being tracked, the density in each cell is given by

$$
\rho=\rho_{\text {oil }} \alpha_{o i l}-\rho_{\text {air }}\left(1-\alpha_{\text {oil }}\right)
$$

All other properties are calculated in the same manner.

The two phases properties are summarized in table 1 .

Table 1: $\quad$ Properties of the two phases at $40^{\circ} \mathrm{C}$.

\begin{tabular}{|c|c|c|}
\cline { 2 - 3 } \multicolumn{1}{c|}{} & $\rho_{40^{\circ} \mathrm{C}}\left[\mathrm{Kg} / \mathrm{m}^{3}\right]$ & $v_{40^{\circ} \mathrm{C}}[\mathrm{Kg} / \mathrm{ms}]$ \\
\hline Air & 1.225 & $1.7894 \mathrm{E}-05$ \\
\hline Lubricant Oil & 1041 & 0.2082 \\
\hline
\end{tabular}

As operating density has been chosen the lowest one.

A single momentum equation is solved throughout the domain, and the resulting velocity field is shared among the phases

$$
\frac{\partial}{\partial t}(\rho \vec{v})+\nabla \cdot(\rho \vec{v} \vec{v})=-\nabla p+\nabla \cdot\left[\mu\left(\nabla \vec{v}+\nabla \vec{v}^{T}\right)\right]+\rho \vec{g}+\vec{F}
$$

One limitation of the shared-fields approximation is that in cases where large velocity differences exist between the phases, the accuracy of the velocities computed near the interface can be adversely affected. The energy equation si also shared among the phases

$$
\frac{\partial}{\partial t}(\rho E)+\nabla \cdot(\vec{v}(\rho E+p))=\nabla \cdot\left(k_{e f f} \nabla T\right)+S_{h}
$$

The VOF model treats energy, $E$, and temperature, $T$, as mass-averaged variables. As with the velocity field, the accuracy of the temperature near the interface is limited in cases where large temperature differences exist between the phases. 
For the pressure-velocity-couplig a SIMPLE scheme has been adopted as suggested for flows in closed domains. This algorithm uses a relationship between velocity and pressure corrections to enforce mass conservation and to obtain the pressure field.

In order to reproduce the operating conditions of the speed reducer, a rigid motion of the mesh has been applied. The motion has been defined by mean of an UdF (User defined Function). All the boundaries has been set to no slip walls: the internal boundaries, corresponding to the planet carrier and planetary gears surfaces, rotate together with the mesh (marked in dark grey in the figure 4) while the other boundaries, corresponding to the external crown and the case of the gearbox (marked in black in the figure 4), are stationary in the absolute reference frame.
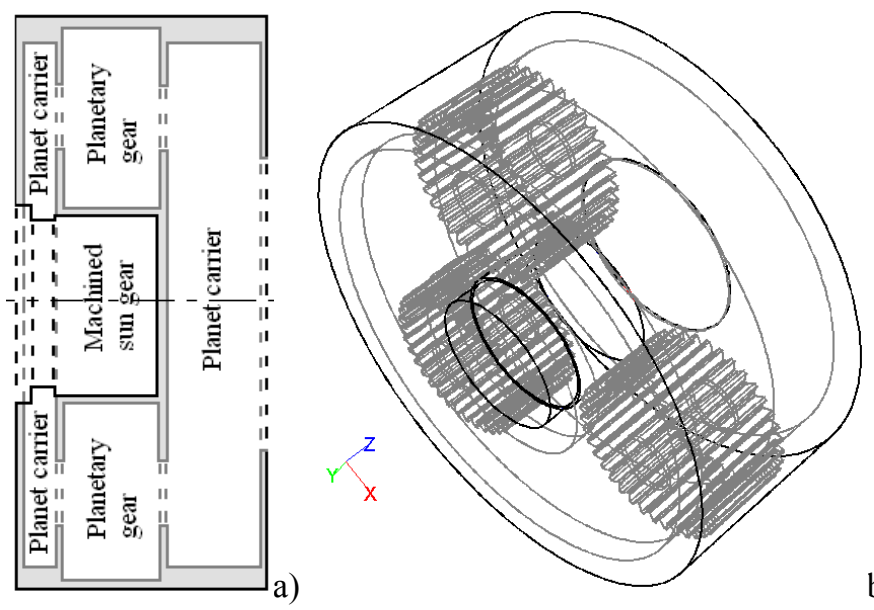

Figure 4: a) 2D schematization of the computational domain (section A-A fig. 3) b) 3D representation of the computational domain.

The time step for the transient analysis has been evaluated as

$$
\Delta t=\frac{V_{\text {cell, } \min }^{\frac{1}{3}}}{U}
$$

where $V_{\text {cell,min }}$ is the volume of the smallest cell in the computational domain and $U$ is the velocity scale of the problem.

\subsection{Operating conditions}

The purpose of the simulations is to analyze the resistant torque on the planet carrier shaft. This moment is calculated with a surface integral on the moving walls with respect to the gearbox axis and it is composed by two parts: the first given by pressure and the second by the viscous effects. Some simulations have been computed with different combinations of oil level, operating temperature and rotational speed. Table 2 shows the combinations of parameters for each 
simulation. $\boldsymbol{T}$ is the operating temperature in ${ }^{\circ} \mathrm{C}, \boldsymbol{\omega}$ the rotational speed of the planet carrier in rpm and $\boldsymbol{L}$ is the oil level in mm measured from the gearbox axis (positive if higher than the axis, negative if lower).

Table 2: $\quad$ Parameters for each simulation.

\begin{tabular}{|c|c|c|}
\hline $\mathrm{T}\left[{ }^{\circ} \mathrm{C}\right]$ & $\mathrm{L}[\mathrm{mm}]$ & $\omega[\mathrm{rpm}]$ \\
\hline 40 & 20 & 500 \\
\hline 40 & 0 & 500 \\
\hline 40 & 0 & 1500 \\
\hline 40 & 20 & 1000 \\
\hline 40 & 20 & 1500 \\
\hline 40 & 0 & 1000 \\
\hline 40 & -20 & 500 \\
\hline 40 & -20 & 1000 \\
\hline 40 & -20 & 1500 \\
\hline 20 & 0 & 1000 \\
\hline 65 & 0 & 1000 \\
\hline
\end{tabular}

The analysis has been stopped after the resistant torque had no more sensible fluctuations and has stabilized. By multiplying the mean value of the torque by the imposed rotational speed, it is possible to determine the churning power losses.
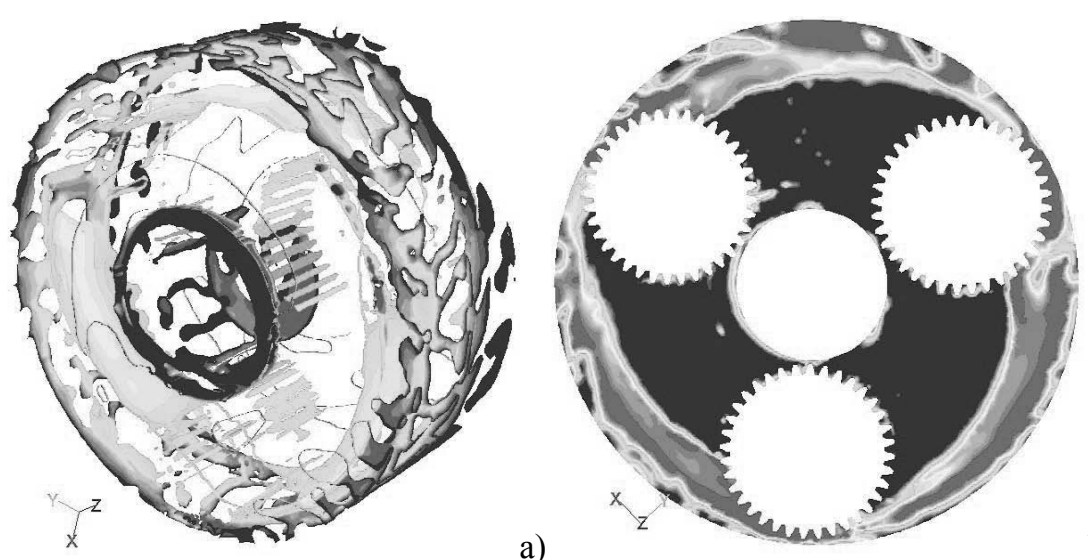

a)

b)

Figure 5: a) Contours of velocity magnitude for the oil phase b) Contours of volume fraction for the oil phase in the mid-section. 


\section{Experimental tests}

In order to provide a validation of the numerical model, a real industrial planetary speed reducer has been modified as already described and tested. After the modifications, the input shaft and the output shaft are completely uncoupled. That allows us to move the planet carrier and reproduce the condition in which the meshing losses and the churning losses due to the rotations of the gears around their axis are avoided.

A schematic layout of the test rig is shown in figure 6. The speed reducer is fixed to the ground by mean of a specially designed structure. The planet carrier shaft is connected to a HBM T12 torque meter with a telemetric transmission of the signal by mean of a double cardan shaft in order to avoid bending. The torque meter, in turn, is connected to a $35 \mathrm{KW}$ DC motor by means of a coupling.

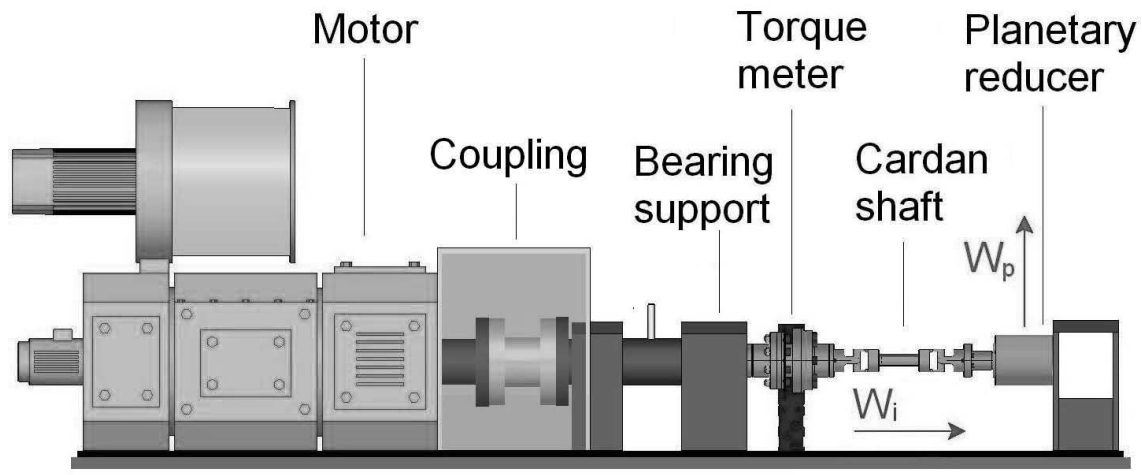

Figure 6: Schematic layout of the test rig.

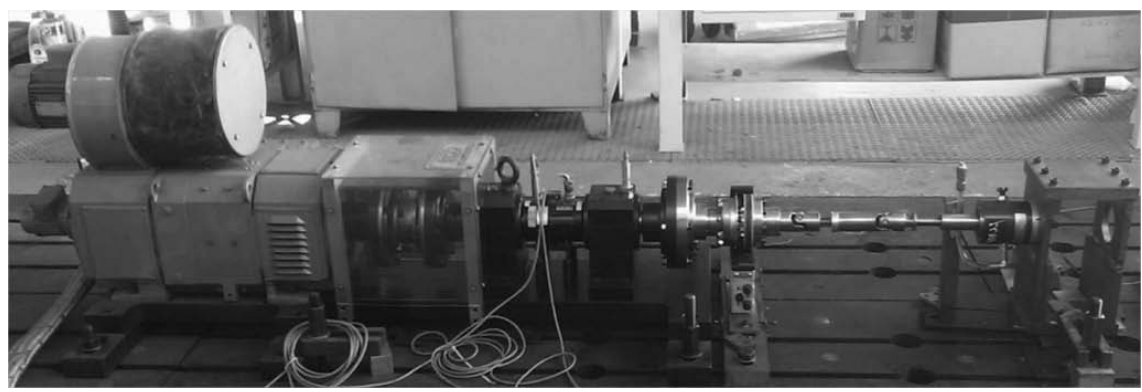

Figure 7: $\quad$ Test rig with the tested speed reducer.

A transparent pipe allows the monitoring of the actual oil level. The operating temperature can be imposed by a special heating system (insulated chamber with a heating source) and measured by mean of a thermocouple. The rotational speed can be controlled by the motor PLC (programmable logic controller). For each of the 3 oil levels, 4 different temperatures have been tested. For all this coupled 
operating conditions (oil level + temperature) the power losses have been measured in a rotational speed range between $100 \mathrm{rpm}$ and $1500 \mathrm{rpm}$.

\section{Results}

Before comparing the experimental results with the computation ones, it is necessary to subtract the seal losses and the bearing losses from the measured values. The torque meter, in fact, measures the input power of the reducer. A little fraction of this power is dissipated by the two internal bearings of the planet carrier shaft and by its seal. This part of losses can be easily calculated by mean of some proved models $[2,3]$ as function of the testing conditions.

Figure 8 to 13 show both the experimental and the computation results for the different oil levels and as a function of temperature and rotational speed.

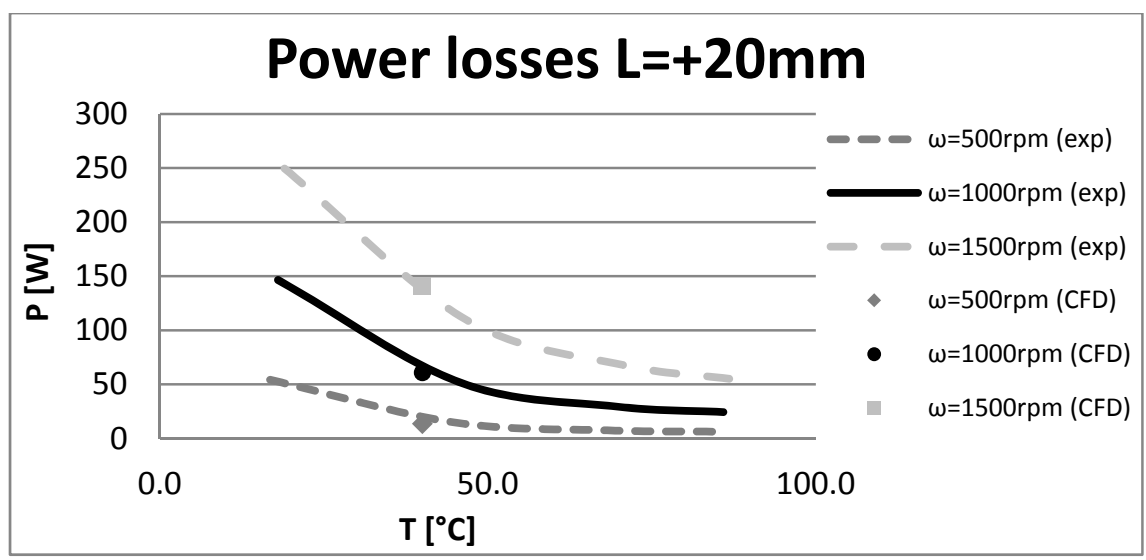

Figure 8: Experimental and computation results for $\mathrm{L}=20 \mathrm{~mm}$.

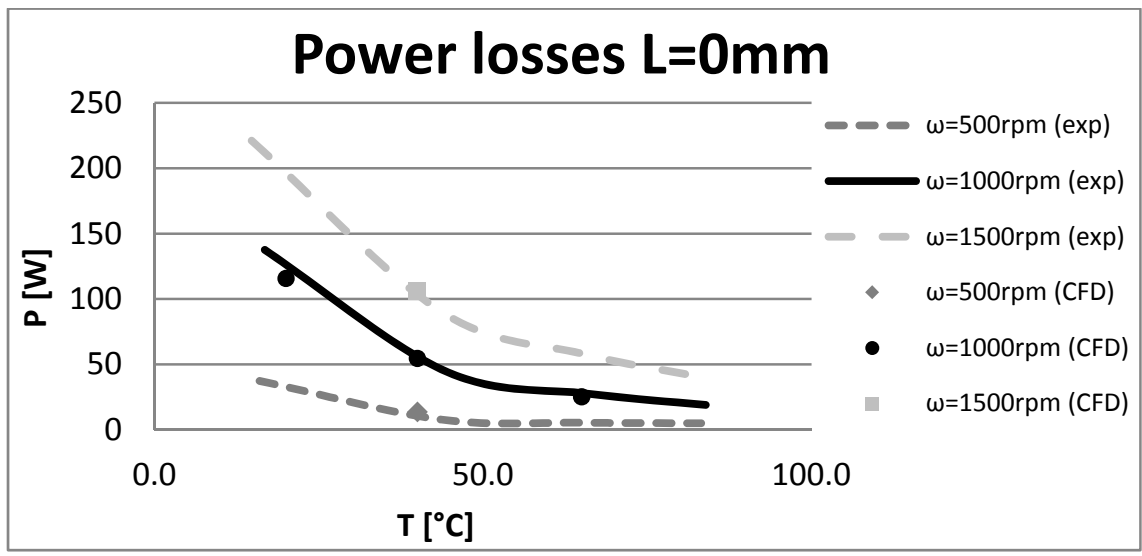

Figure 9: Experimental and computation results for $\mathrm{L}=0 \mathrm{~mm}$. 


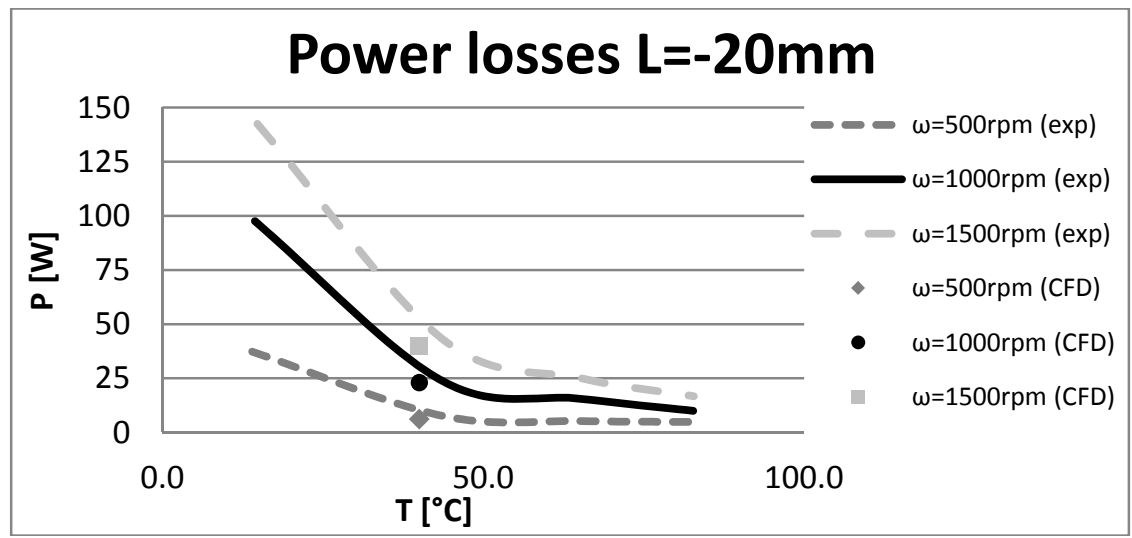

Figure 10: Experimental and computation results for $\mathrm{L}=-20 \mathrm{~mm}$.

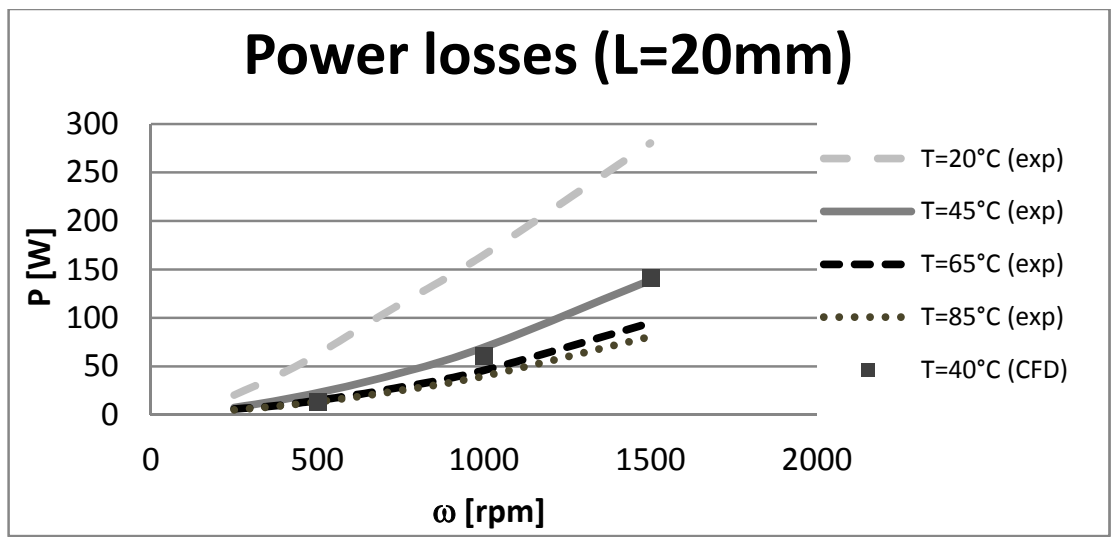

Figure 11: Experimental and computation results for $\mathrm{L}=20 \mathrm{~mm}$.

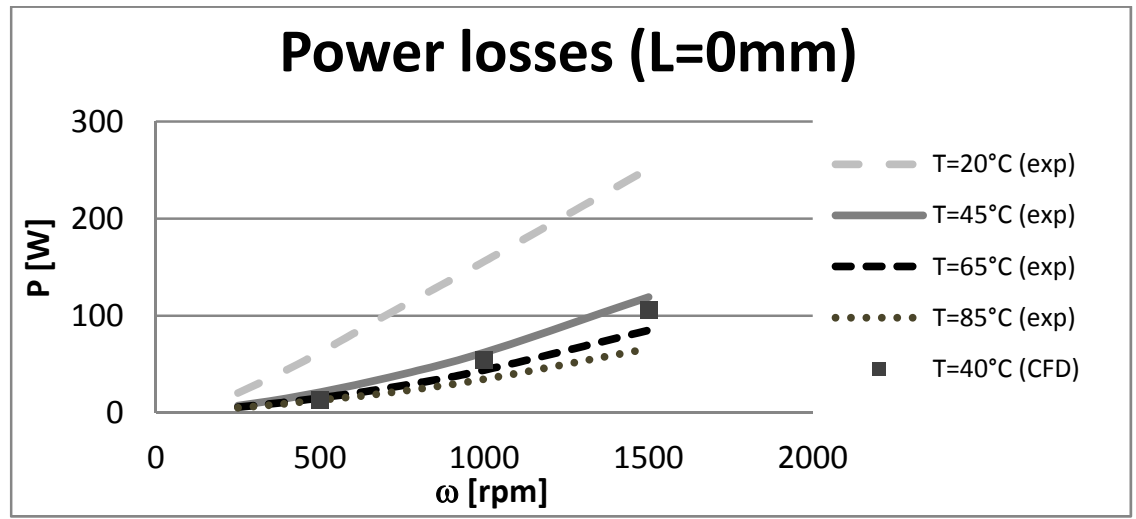

Figure 12: Experimental and computation results for $\mathrm{L}=0 \mathrm{~mm}$. 


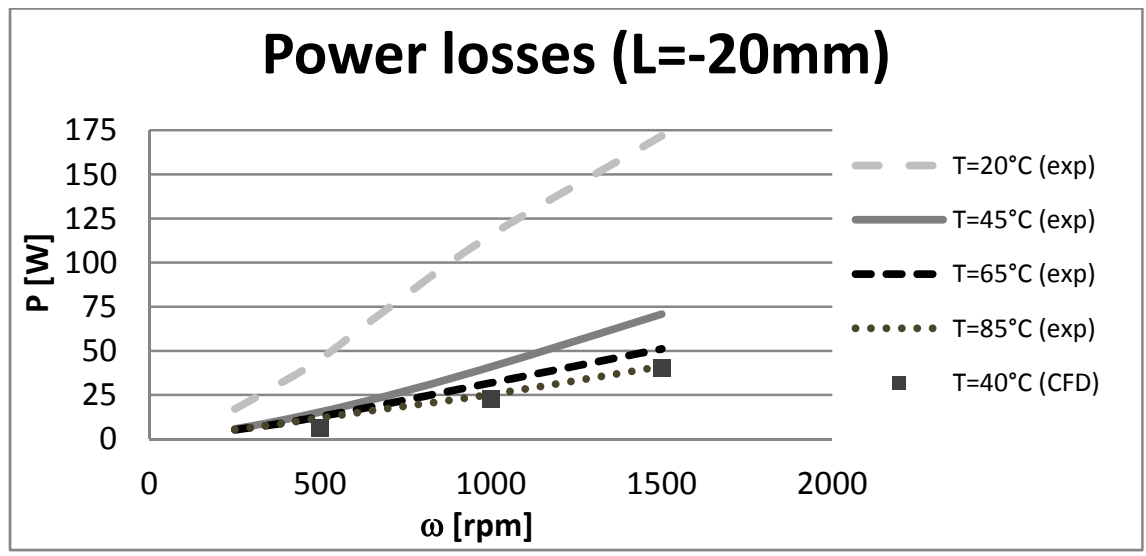

Figure 13: Experimental and computation results for $\mathrm{L}=-20 \mathrm{~mm}$.

Due to the high computational time needed for the solution of the numerical models, not all the experimentally tested conditions have been also computed with the CFD analysis.

It can be seen that the numerical results are in good agreement with the numerical ones.

\section{Conclusions}

As reliable models to predict the churning losses of the planetary gears are still not available, a CFD model has been applied in order to predict this important component of losses of epicycloid gear reducers, starting from the geometry and the operating conditions. The results of the model are well supported by the experiments (except for extremely low temperatures). The losses increase, as expected, with static lubricant level and rotational speed and decrease with the temperature. The decrease rate of the power losses with the temperature is very high for low temperature and decreases with the temperature growth.

The increase rate of the losses with the rotational speed is more than linear.

Future development is the investigation of other influence parameters like the oil type and the geometry of the reducer.

\section{References}

[1] Concli, F., Gorla, C., Arigoni, R., Cognigni, E., Musolesi, M., Planetary Speed Reducers: Efficiency, Backlash, Stiffness, International conference on gears, Munich 2010.

[2] General Catalogue SKF — SKF Group, December 2006.

[3] Niemann, G., Winter, H., Maschinenelemente - Band 2: Getriebe Allgemein, Zahnradgetriebe - Grundlagen, Stirnradgetriebe - 2. Auflage , Springer, Berlin 2003. 
[4] ISO/TR 14179-1 and -2.

[5] Patankar, S.V., Numerical heat transfer and fluid flow, Taylor\&Francis, USA 1980.

[6] Versteeg, H.K., Malalasekera, W., An introduction to computational fluid dynamics - The finite volume method, Longman Group, London 1995.

[7] Comini, G., Fondamenti di termofluidodinamica computazionale, SGEditoriali, Padova 2004.

[8] Concli, F., Gorla, C., Arigoni, R., Musolesi, M., Riduttori di precisione a gioco ridotto ed alta efficienza, Organi di trasmissione - febbraio 2011, Tecniche Nuove, Milano 2011.

[9] Csobàn, A., Kozma, M., Influence of the Oil Churning, the Bearing and the Tooth Friction Losses on the Efficiency of Planetary Gears, Journal of Mechanichal Engineering 56(2010)4, pp.231-238. 